\title{
Using SNP markers to dissect linkage disequilibrium at a major quantitative trait locus for resistance to the potato cyst nematode Globodera pallida on potato chromosome V
}

\author{
Ute Achenbach $\cdot$ Joao Paulo $\cdot$ Evgenyia Ilarionova $\cdot$ \\ Jens Lübeck • Josef Strahwald • Eckhard Tacke • \\ Hans-Reinhard Hofferbert $\cdot$ Christiane Gebhardt
}

Received: 26 June 2008 / Accepted: 24 October 2008 / Published online: 20 November 2008

(c) The Author(s) 2008. This article is published with open access at Springerlink.com

\begin{abstract}
The damage caused by the parasitic root cyst nematode Globodera pallida is a major yield-limiting factor in potato cultivation. Breeding for resistance is facilitated by the PCR-based marker 'HC', which is diagnostic for an allele conferring high resistance against $G$. pallida pathotype $\mathrm{Pa} 2 / 3$ that has been introgressed from the wild potato species Solanum vernei into the Solanum tuberosum tetraploid breeding pool. The major quantitative trait locus (QTL) controlling this nematode resistance maps on potato chromosome $\mathrm{V}$ in a hot spot for resistance to various pathogens including nematodes and the oomycete Phytophthora infestans. An unstructured sample of 79 tetraploid, highly heterozygous varieties and breeding clones was selected based on presence (41 genotypes) or absence (38 genotypes) of the HC marker. Testing the clones for resistance
\end{abstract}

Communicated by J. E. Bradshaw.

Electronic supplementary material The online version of this article (doi:10.1007/s00122-008-0925-x) contains supplementary material, which is available to authorized users.

U. Achenbach $\cdot$ J. Paulo $\cdot$ E. Ilarionova $\cdot$ C. Gebhardt $(\bowtie)$

Department of Plant Breeding and Genetics,

Max Planck Institute for Plant Breeding Research,

50829 Cologne, Germany

e-mail: gebhardt@mpiz-koeln.mpg.de

J. Lübeck · J. Strahwald

Saka-Pflanzenzucht GbR, Zuchtstation Windeby,

24340 Windeby, Eckernförde, Germany

E. Tacke

Bioplant GmbH, 29574 Ebstorf, Germany

H.-R. Hofferbert

Böhm-Nordkartoffel Agrarproduktion GbR,

29574 Ebstorf, Germany to G. pallida confirmed the diagnostic power of the $\mathrm{HC}$ marker. The 79 individuals were genotyped for 100 single nucleotide polymorphisms (SNPs) at 10 loci distributed over $38 \mathrm{cM}$ on chromosome V. Forty-five SNPs at six loci spanning $2 \mathrm{cM}$ in the interval between markers GP21GP179 were associated with resistance to G. pallida. Based on linkage disequilibrium (LD) between SNP markers, six LD groups comprising between 2 and 18 SNPs were identified. The LD groups indicated the existence of multiple alleles at a single resistance locus or at several, physically linked resistance loci. LD group C comprising 18 SNPs corresponded to the 'HC' marker. LD group E included 16 SNPs and showed an association peak, which positioned one nematode resistance locus physically close to the $R I$ gene family.

Present Address:

U. Achenbach

Rheinische Friedrich-Wilhelms-Universität Bonn,

Institute for Molecular Physiology and Biotechnology

of Plants (IMBIO), 53115 Bonn, Germany

Present Address:

J. Paulo

Biometris, Wageningen University,

6700 AC Wageningen, The Netherlands

Present Address:

E. Ilarionova

DNA LandMarks Inc., 84 rue Richelieu,

St Jean-sur-Richelieu, QC J3B 6X3, Canada 


\section{Introduction}

The damage caused by the parasitic root cyst nematode Globodera pallida (Greco 1988; Mugniéry and Phillips 2007 ) is a major yield-limiting factor in potato cultivation. Under poor nematode management, yield losses induced by G. pallida can range from 20 to $70 \%$. The cultivation of nematode-resistant varieties is a solution to this problem. The introgression and maintenance of resistance against $G$. pallida in the breeding pool is therefore an important goal in the development of new potato varieties. Due to its quantitative nature, the phenotypic evaluation of resistance to $G$. pallida is time consuming, costly and often ambiguous. DNA markers could facilitate breeding for resistance, when they are diagnostic for favourable alleles and thus allow for a precise and efficient selection of resistant clones at an early stage in the selection process.

Molecular mapping has repeatedly revealed a major quantitative resistance locus (QRL) against $G$. pallida on potato chromosome V (Kreike et al. 1994; Rouppe van der Voort et al. 1998, 2000; Caromel et al. 2003; Sattarzadeh et al. 2006). This QRL is tagged by the DNA markers GP21 and GP179 flanking a genetic interval of $3 \mathrm{cM}$ (Meksem et al. 1995). This region of the potato genome is known as a hot spot for qualitative and quantitative resistance factors to various pathogens (reviewed in Gebhardt and Valkonen 2001). One of the resistance factors in the GP21-GP179 interval is the $R 1$ gene for resistance to Phytophthora infestans causing late blight, which is a member of a clustered gene family having a CC (coiled coil), a NB (nucleotide binding) and a LRR (leucine rich repeat) domain (Ballvora et al. 2007). Physically close to the $R I$ locus, in fact, within one of the $R l$ family members, maps the PCR-based marker 'HC'. The HC marker was developed based on SNPs (single nucleotide polymorphisms) linked to the G. pallida QRL and was shown to be diagnostic for high resistance to G. pallida pathotypes $\mathrm{Pa} / 3$ in modern potato varieties (Sattarzadeh et al. 2006). The strong association of the $\mathrm{HC}$ marker with resistance to $G$. pallida may result from $\mathrm{HC}$ being located in the resistance gene itself, or, more likely, HC being physically separated from, but in strong linkage disequilibrium (LD) with the resistance gene(s). In the latter case, $\mathrm{HC}$ is part of a haplotype block of unknown size.

Molecular identification of the gene(s) underlying the $G$. pallida QRL requires increased genetic resolution, which can be achieved by generating a large segregating population and analysing it for rare recombination events flanking the target locus. Alternatively, historical recombination events in contemporary germplasm may be analysed, which have occurred during the meiotic cycles since the resistance was introgressed into modern potato varieties from the wild, tuber-bearing species Solanum vernei (Sattarzadeh et al.
2006). In the absence of population substructure, the estimator for the genetic distance between a marker and the targeted resistance locus is given by the amount of linkage disequilibrium (LD), that is, the non-random association of alleles at two or more loci (Tenesa et al. 2003). In populations of individuals related by descent, marker loci that are in LD with each other may show a gradient of the strength of their association with a QTL, thereby permitting QTL fine mapping. SNPs, which are found with high frequency in amplicons generated from genomic DNA of heterozygous, diploid and tetraploid individuals (Rickert et al. 2003; Simko et al. 2006) are most informative about LD analysis in potato. Moreover, SNPs allow estimating the allele dosage in tetraploid individuals (Rickert et al. 2003). Haplotypes, which are particular combinations of alleles at physically linked loci, can be more informative than individual SNPs. In potato, the inference of haplotypes from sequence data is complicated because SNP data are unphased due to the heterozygocity of individual genotypes.

In this paper, we describe the results of genotyping an unstructured population of tetraploid varieties and breeding clones with and without the HC marker for 100 SNPs at ten loci on potato chromosome $\mathrm{V}$. The aim of our study was (1) to estimate the size of the haplotype block around the $\mathrm{HC}$ marker and (2) to test whether LD analysis reveals more precisely the position of the gene(s) underlying the major QRL to G. pallida on chromosome V.

\section{Materials and methods}

Plant material

Eighteen varieties and 189 tetraploid clones from breeding programs were provided by Saka-Ragis Pflanzenzucht (SaRa), Böhm-Nordkartoffel Agrarproduktion (BNA), Bavaria Saat (by courtesy of A. von Zwehl), HZPC Holland B.V., Averis Seeds B.V., Karna, E.J. Feunekes, Kweekbedrijf Prummerl and B.F. Leestemaker \& A. Smid (Table 1). The clones from Saka-Ragis Pflanzenzucht and BöhmNordkartoffel Agrarproduktion are identified with ' $\mathrm{S}$ ' and ' $\mathrm{B}$ ', respectively, followed by a running number from 1 to 120. The $\mathrm{S}$ and $\mathrm{B}$ clones were sampled from the two companies conducting breeding programs for resistance to $G$. pallida and late blight, excluding full sib clones. The clones represented a broad range of breeding materials.

\section{Assessment of resistance to G. pallida}

Pot inoculation tests were carried out according to Kort et al. (1977). The resistance phenotype was quantified as the average number of newly formed cysts per pot. The BNA clones were tested once with two to three replicates 
Table 1 Globodera pallida resistance phenotypes of the HC positive sample population and the $\mathrm{HC}$ negative control population

\begin{tabular}{|c|c|c|c|}
\hline \multicolumn{2}{|c|}{$\mathrm{HC}$ marker positive } & \multicolumn{2}{|c|}{$\mathrm{HC}$ marker negative } \\
\hline Genotype & Phenotype & Genotype & Phenotype \\
\hline Avano & Resistant to $\mathrm{Pa} 2 / 3^{\mathrm{a}}$ & Elles & Resistant to $\mathrm{Pa} 2$ \\
\hline Avarna & Resistant to $\mathrm{Pa} 2 / 3$ & Feska & Resistant to $\mathrm{Pa} 2$ \\
\hline Averia & Resistant to $\mathrm{Pa} 2 / 3$ & Kantara & Partial res. to $\mathrm{Pa} 2 / 3$ \\
\hline Aviala & Resistant to $\mathrm{Pa} 2 / 3$ & Karida & Resistant to $\mathrm{Pa} 2$ \\
\hline Brisant & Resistant to $\mathrm{Pa} 2 / 3$ & Karnico & Resistant to $\mathrm{Pa} 2$ \\
\hline Festien & Resistant to $\mathrm{Pa} 2 / 3$ & Katinka & Partial res. to $\mathrm{Pa} 2 / 3$ \\
\hline Innovator & Resistant to $\mathrm{Pa} 2 / 3$ & Pallina & Partial res. to $\mathrm{Pa} 2 / 3$ \\
\hline Karakter & Resistant to $\mathrm{Pa} 2 / 3$ & Producent & Resistant to $\mathrm{Pa} 2$ \\
\hline Melanie & Resistant to $\mathrm{Pa} 2 / 3$ & $B 01$ & $97^{\mathrm{b}}$ \\
\hline Sjamero & Resistant to $\mathrm{Pa} 2 / 3$ & BO2 & 80 \\
\hline$B 23$ & 5 & $B 03$ & 146 \\
\hline$B 24$ & 3 & B04 & 57 \\
\hline$B 25$ & 15 & B05 & 107 \\
\hline B37 & 2 & B06 & 92 \\
\hline B39 & 0 & $B 07$ & 87 \\
\hline$B 41$ & 0 & B08 & 165 \\
\hline B42 & 2 & $B 09$ & 73 \\
\hline$B 43$ & 0 & $B 10$ & 48 \\
\hline B44 & 0 & $B 11$ & 67 \\
\hline$B 47$ & 29 & $B 12$ & 42 \\
\hline B48 & 8 & $B 13$ & 91 \\
\hline B50 & 1 & $B 14$ & 132 \\
\hline B51 & 1 & $B 15$ & 64 \\
\hline$B 52$ & 10 & $B 16$ & 75 \\
\hline B55 & 6 & $B 17$ & 80 \\
\hline$B 80$ & 0 & $B 18$ & 160 \\
\hline B96 & 1 & $B 19$ & 113 \\
\hline B108 & 0 & B31 & 33 \\
\hline $\mathrm{SO} 2$ & 2 & $S 15$ & 137 \\
\hline $\mathrm{SO} 3$ & 0 & $S 16$ & 120 \\
\hline SO7 & 2 & $S 17$ & 88 \\
\hline SO8 & 6 & $S 18$ & 165 \\
\hline SO9 & 0 & $S 19$ & 144 \\
\hline$S 28$ & 0 & $S 20$ & 108 \\
\hline S5O & 1 & & \\
\hline S61 & 0 & & \\
\hline$S 73$ & 1 & & \\
\hline$S 83$ & 2 & & \\
\hline$S 101$ & 0 & & \\
\hline S105 & 2 & & \\
\hline$S 108$ & 1 & & \\
\hline
\end{tabular}

${ }^{a}$ According to variety passport data (Sattarzadeh et al. (2006))

b Average number of newly formed cysts (rounded)

per genotype at the 'Landwirtschaftskammer Niedersachsen' (Hannover, Germany) using for inoculation the $G$. pallida population 'Kalle' (pathotype Pa2). The SaRa clones were evaluated in 3 years (2004, 2005 and 2006) with four replicates per genotype at the 'Landesamt für Landwirtschaft, Lebensmittelsicherheit und Fischerei Mecklenburg-Vorpommern' (Rostock, Germany). Here the 'Chavornay' population (pathotype $\mathrm{Pa} 2 / 3$ ) was used for inoculation. The G. pallida resistance phenotype of the varieties was obtained from the variety lists of the Netherlands (81e rassenlijst Landbouwgewassen, 2006, ISSN 0168-7484) and Germany (Beschreibende Sortenliste Kartoffeln, 2005, ISSN 1430-9777).

\section{DNA isolation}

Young, healthy potato leaves were harvested from each individual, freeze dried and stored in air-tight containers at $-20^{\circ}$. Total genomic DNA was extracted from 0.3 to $0.4 \mathrm{~g}$ freeze dried leaf material according to Bormann et al. (2004).

SNP (single nucleotide polymorphism) genotyping

Amplicons were generated from $50 \mathrm{ng}$ genomic DNA as template in $20 \mu \mathrm{l}$ buffer $(20 \mathrm{mM}$ Tris- $\mathrm{HCl}, \mathrm{pH} 8.4,2.5 \mathrm{mM}$ $\mathrm{MgCl}_{2}, 50 \mathrm{mM} \mathrm{KCl}$ ), including $0.25 \mu \mathrm{M}$ of each primer (Table 2), $0.2 \mathrm{mM}$ dNTP and 1.0 unit Taq DNA polymerase (Invitrogen Life Technologies, Freiburg, Germany). Standard cycling conditions were $3 \mathrm{~min}$ initial denaturation at $94^{\circ}$, followed by 39 cycles of 1 min denaturation at $94^{\circ}$, $1 \mathrm{~min}$ annealing at the appropriate $T_{\mathrm{m}}$ (Table 2) and $1 \mathrm{~min}$ extension at $72^{\circ}$. Reactions were finished by 8 min incubation at $72^{\circ}$. PCR products were examined for quality on ethidium bromide stained agarose gels. Prior to sequencing, PCR products were purified with ExoSAP-IT ${ }^{\circledR}$ (USB Corporation, Cleveland, USA) according to supplier's instructions. Amplicons were custom sequenced at the MPIZ DNA core facility using an Applied Biosystems Abi Prism 3730 sequencer (Weiterstadt, Germany) and BigDye-terminator v3.1 chemistry. Premixed reagents were from Applied Biosystems. Oligonucleotides were purchased from Invitrogen, Life Technologies (Freiburg, Germany) or QIAGEN GmbH (Hilden, Germany). Sequencing data of all individuals were aligned using the multalin interface page (Corpet 1988) to detect SNP positions. For estimating the dosage of SNP alleles in heterozygous, tetraploid individuals $(3: 1,2: 2$ or $1: 3)$, the sequence tracefiles were subjected to the Data Acquisition and Analysis Software (DAx) (http://www.dax.nl/daxapp1.htm).

SSR (simple sequence repeat) genotyping

A set of 19 SSR marker loci with a random genome wide distribution were genotyped to be used in the analysis of population substructure. The following 19 SSR-marker loci 
Table 2 Loci analysed for SNPs, accession number or reference, oligonucleotides used for PCR amplification and amplicon sequencing, amplicon size, and annealing temperature

\begin{tabular}{|c|c|c|c|c|}
\hline Locus & Accession number, reference & Primer sequence $\left(5^{\prime}-3^{\prime}\right)^{\mathrm{a}}$ & Amplicon size (bp) & $T_{\mathrm{A}}\left({ }^{\circ} \mathrm{C}\right)$ \\
\hline$G P 21$ & $\begin{array}{l}\text { AJ487347, } \\
\text { Meksem et al. (1995) }\end{array}$ & $\begin{array}{l}\text { f-CCСTGAACCTCCTTATCCCC } \\
\text { r,s-ACTATTATGTCTATGAGG AAG TGG TC }\end{array}$ & 754 & 61 \\
\hline ORF3 & EF514212 & $\begin{array}{l}\text { f-TTGCTTGAACATGATCCACAC } \\
\text { r-GCTTAGTTTCCTCTGGCACC } \\
\text { f,s-CGAGAAGAGGAAAAAGATGTC } \\
\text { f,s-GCTATGAACTTCATCAACATG }\end{array}$ & 750 & 59 \\
\hline ORF 12 & EF514212 & $\begin{array}{l}\text { f,s-CGATGAATATGAAGATGAAGC } \\
\text { r-ACCTTTTTAGCTTTGATACGTTG }\end{array}$ & 426 & 57 \\
\hline ORF20 & EF514212 & $\begin{array}{l}\text { f,s-ACAAGTACAGCTAATAGACCC } \\
\text { r-GGCTTATCGTCTTCACTACC }\end{array}$ & 558 & 55 \\
\hline$B A 87 d 17 t 3$ & CG782997 & $\begin{array}{l}\text { f-GTAGTACATCAACATACATTTTGCGG } \\
\text { r-CTCAGAATTCAGAGCTTCAACTGATG } \\
\text { f,s-AACAGGCTTAATCCTCATCCGC }\end{array}$ & 548 & 56 \\
\hline$B A 213 c 14 t 7$ & CG783029 & $\begin{array}{l}\text { f,s-CAATTGATTCATTTTATGTAGCGAG } \\
\text { r-CTTGACGCAAACCTCTGCGAG }\end{array}$ & 559 & 56 \\
\hline$P R K$ & AY730335 & $\begin{array}{l}\text { f,s-ACTTGAAATGATAACAGGTAGGAG } \\
\text { r-CCСTTTTGCCAGTTCATCC }\end{array}$ & 550 & 58 \\
\hline GP179 & AY730335 & $\begin{array}{l}\text { f-TCCTCCTTTGAAATATGTTTCACTTCTA } \\
\mathrm{r}^{\text {- }}{ }^{\text {biotin }} \text { CCCATGGCTACATGCATTATGTATT } \\
\text { s-ACTTCTAAGTGATAATCTTGA }\end{array}$ & 186 & 56 \\
\hline StPto & $\mathrm{CK} 256750^{\mathrm{b}}$ & $\begin{array}{l}\text { f,s-TCACATTGGATTGGGTGGC } \\
\text { r-CGAGTCCACTGCCCATTC }\end{array}$ & 670 & 55 \\
\hline 239E4left & Bakker et al. (2004) & $\begin{array}{l}\text { f,s-GGCCCCACAAACAAGAAAAC } \\
\text { r-AGGTACСТССАТСТССАТTTTGTAAG }\end{array}$ & 340 & 56 \\
\hline
\end{tabular}

\footnotetext{
${ }^{a}$ Forward primers are indicated with $\mathrm{f}$, reverse primers with $\mathrm{r}$, primers used for amplicon sequencing with s. The ORF3 amplicon was sequenced with two different forward primers (f, $s$ ) to circumvent Indels

$\mathrm{r}^{\text {biotin }}$ was the biotinylated primer used for pyrosequencing

b Potato EST with 91\% nucleotide identity to the tomato Pto gene Martin et al. (1993)
}

were analysed: StIO04, StI013, StIO20, StIO22, StI023, StIO24, StIO28, StIO43, StIO47 and StIO58 according to Feingold et al. 2005; STM0037, STM0038, STM1043, STM1052, STM1097, STM1104, STM1106, STM2012 and STM3012 according to Milbourne et al. 1998. SSR alleles were size separated on SPREADEX gels (Elchrom Scientific, Cham, Switzerland) using the SEA 2000 Electrophoresis System (Elchrom Scientific) according to manufacturer's instructions. Gels were stained after electrophoresis with $10 \%$ SYBR ${ }^{\mathrm{TM}}$ Gold in $30 \mathrm{mM}$ TAE buffer for $45 \mathrm{~min}$ and de-stained for $30 \mathrm{~min}$ in destaining Solution (Elchrom Scientific) in $12 \mathrm{mM}$ TAE. Two to seven alleles were scored per SSR locus. Allele dosage (quadruplex, triplex, duplex, simplex or nulliplex) was estimated based on the number of alleles per individual and the band intensity of the alleles present in one individual relative to each other.
Pyrosequencing

The marker GP179 was analysed by pyrosequencing (Biotage AB, Uppsala, Sweden) according to the manufacturer's instructions. A fragment of 186 base pairs was amplified using the primer combination GP179r- ${ }^{\text {biotin }}$ and GP179f (Table 2). PCR was carried out in a total volume of $25 \mu \mathrm{l}$ containing $4 \mathrm{mM}$ Tris- $\mathrm{HCl}, \mathrm{pH} 8.3,20 \mathrm{mM} \mathrm{KCl}$, $3 \mathrm{mM} \mathrm{MgCl} 2,0.5 \mu \mathrm{M}$ each primer, $0.2 \mathrm{mM}$ dNTPs, 1 unit Taq DNA Polymerase (Invitrogen Life Technologies, Freiburg, Germany). After initial denaturation at $94^{\circ}$ for $2 \mathrm{~min}$, 50 cycles of amplification were carried out starting at $93^{\circ}$ for $45 \mathrm{~s}$, followed by $45 \mathrm{~s}$ at $57^{\circ}$ and $1 \mathrm{~min}$ at $72^{\circ}$, with a final extension at $75^{\circ}$ for $10 \mathrm{~min}$. PCR products were quantified on $1.5 \%$ agarose gels relative DNA standards. The pyrosequencing reactions were performed automatically with a PSQ 96 system (Biotage AB, Uppsala, Sweden) 
using a SNP reagent kit according to the manufacturer's instructions and the sequencing primer GP179s (Table 2). All oligonucleotides were custom synthesized and HPLCpurified by SIGMA-Genosys, Germany.

\section{Data analysis}

Population structure was evaluated by the program STRUCTURE version 2.1 (Pritchard et al. 2000) using the genetic information provided by 113 SSR alleles at 19 loci with a genome wide distribution. The number $k$ of underlying subpopulations was set to vary between two and ten. For each $k$ value, two runs of 600,000 iterations were performed, of which the first 100,000 were discarded as burn in. An admixture model with independent allele frequencies was assumed, without a priori population information.

The degree of linkage disequilibrium (LD) between pairs of SNP markers was based on a statistical test of independence, assuming no population substructure. In tetraploid individuals, each bi-allelic SNP marker occurs in one of five genotype classes: $1 / 1 / 1 / 1,1 / 1 / 1 / 2,1 / 1 / 2 / 2,1 / 2 / 2 / 2$ or $2 / 2 / 2 /$ 2 , with 1 and 2 corresponding to alternative nucleotide alleles. The genotype classes for each SNP locus were first defined by the observed combination and dosage of nucleotides. Then, tests for independence of pairs of SNP markers were performed on the frequencies of genotype-class combinations, using the Exact Test for RxC tables, available in SAS Proc Freq (SAS Institute Inc. 2004). The $p$-values resulting from testing all SNP pairs were a posteriori corrected for multiple testing, according to the Two-Stage Linear Step-Up Procedure (Benjamini et al. 2005), and the values after the correction ( $q$-values) used as a measure of the degree of independence, so that a lower $q$-value corresponds to larger evidence against independence of a pair of SNPs. A threshold of 0.01 was taken for the false discovery rate.

Associations between SNP markers and nematode resistance phenotype were analysed with Fisher's exact test on $2 \times 2$ contingency tables using SPSS (SPSS 13 for Windows, SPSS Inc. Chicago, IL, USA).

\section{Results}

A total of 189 tetraploid-breeding clones were evaluated for presence of the $\mathrm{HC}$ marker. Thirty-one clones were $\mathrm{HC}$ positive. These and $10 \mathrm{HC}$ positive varieties (Sattarzadeh et al. 2006) were selected as a sample population of 41 individuals having the $\mathrm{HC}$ marker. The control population negative for the HC marker consisted of 30 breeding clones and 8 varieties (Table 1). The status for resistance to $G$. pallida of the varieties was known (Table 1). When the ' $\mathrm{S}$ ' clones were tested for resistance to G. pallida with the 'Chavornay' population (pathotype Pa2/3), all HC-positive clones were highly resistant (on average 0-6 newly formed cysts), whereas the HC-negative clones were susceptible (between 30 and 200 newly formed cysts), thus confirming the diagnostic power of the HC assay (Table 1). The HCpositive ' $\mathrm{B}$ ' clones were therefore predicted to be resistant to $G$. pallida pathotype $\mathrm{Pa} 2 / 3$. When tested for resistance to G. pallida with population 'Kalle' (pathotype Pa2), the HCpositive ' $\mathrm{B}$ ' clones were highly resistant $(0-10$ newly formed cysts) except clones $B 25$ and $B 47$, which were slightly susceptible (average cyst counts 15 and 29)(Table 1). The phenotypes of $B 25$ and $B 47$ may result from true recombination events or from errors in the resistance tests. The HC-negative ' $\mathrm{B}$ ' clones were all susceptible (between 30 and 160 newly formed cysts) (Table 1).

Ten loci spanning a genetic distance of approximately $38 \mathrm{cM}$ on chromosome V (Meksem et al.1995; Meyer et al. 2005; Niewöhner et al. 1995) were scored for SNPs in the 79 individuals (Table 2, Fig. 1, sequences in supplementary material). The loci ORF3, ORF12, ORF20, BA87d17t3 and $B A 213 c 14 t 7$ were part of a contiguous genomic sequence of 417 kilobase pairs located in the $3 \mathrm{cM} \mathrm{GP21-GP179}$ interval (Ballvora etal. 2007). The loci BA87d17t3 and $B A 213$ c $14 t 7$ are insertion ends of the BAC clones BA $87 \mathrm{~d} 17$ and BA213c14 and have been previously analysed in the QRL linkage study of Sattarzadeh et al. (2006). BA87d17t3 contains the two SNPs (BA87d17t3-SNP212, BA87d17t3SNP444) used to design the HC marker (Sattarzadeh et al. 2006). The loci $P R K$ and GP179 were part of a $41-\mathrm{kbp}$ genomic sequence of BAC clone BA151m8 (gene bank accession AY730335). The loci StPto (potato homolog of the tomato Pto gene for resistance to Pseudomonas syringae (Martin et al. 1993, Leister et al. 1996) and 239E4left, tightly linked to the $\mathrm{Hl}$ gene for resistance to the root cyst nematode G. rostochiensis (Niewöhner et al. 1995, Bakker et al. 2004), were located outside the GP21-GP179 interval. In addition, 113 SSR alleles at 19 loci with a genome wide distribution were genotyped to assess population substructure. The likelihood of the data, conditional on the assumed number $k$ of underlying subpopulations always increased with $k$, supporting the hypothesis of an unstructured population. Furthermore, in the model assuming population admixture we found that for $k>2$ no individual was allocated to a single group; instead, individuals' ancestry was

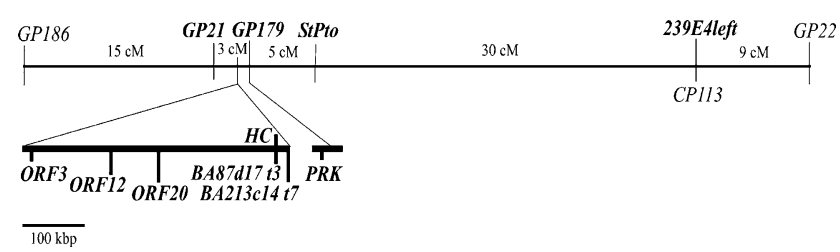

Fig. 1 Genetic and physical map of potato chromosome V. The ten loci analysed for SNPs are shown in bold print. The $H C$ Marker was derived from 2 SNPs in the BA87d17t3 amplicon 
Table 3 Number of SNPs scored, and SNPs associated with resistance to G. pallida at $P<0.01$

\begin{tabular}{|c|c|c|c|}
\hline Locus & $\begin{array}{l}\text { No of SNPs } \\
\text { scored }\end{array}$ & $\begin{array}{l}\text { No of SNPs } \\
\text { associated }\end{array}$ & Position $^{\text {a }}$ (SNP alleles) of the associated SNPs \\
\hline$G P 21$ & 17 & 10 & $\begin{array}{l}1090(\mathrm{~A} / \mathrm{C}), \mathbf{1 1 4 4}(\mathrm{A} / \mathrm{T}), \mathbf{1 1 5 5}(\mathrm{A} / \mathrm{G}), \mathbf{1 1 5 9}(\mathrm{A} / \mathrm{G}), \mathbf{1 1 6 5}(\mathrm{C} / \mathrm{G}), \mathbf{1 1 7 0}(\mathrm{C} / \mathrm{T}), \mathbf{1 1 8 5}(\mathrm{C} / \mathrm{T}) \\
1199(\mathrm{~A} / \mathrm{T}), \mathbf{1 2 5 7}(\mathrm{G} / \mathrm{T}), \mathbf{1 2 7 4}(\mathrm{A} / \mathrm{T})\end{array}$ \\
\hline ORF3 & 20 & 12 & $\begin{array}{l}\text { 93( } \mathrm{C} / \mathrm{T}), \mathbf{1 4 3}(\mathrm{C} / \mathrm{T}), \mathbf{3 2 8}(\mathrm{A} / \mathrm{G}), \mathbf{3 8 0}(\mathrm{A} / \mathrm{G}), \mathbf{4 3 3}(\mathrm{A} / \mathrm{C}), \mathbf{4 3 8}(\mathrm{C} / \mathrm{G}), \mathbf{4 6 0}(\mathrm{C} / \mathrm{G}), \mathbf{4 7 2}(\mathrm{A} / \mathrm{T}), \\
\mathbf{5 0 3}(\mathrm{C} / \mathrm{T}), \mathbf{5 2 0}(\mathrm{A} / \mathrm{G}), \mathbf{5 9 6}(\mathrm{A} / \mathrm{T}), \mathbf{6 1 1}(\mathrm{A} / \mathrm{G} / \mathrm{T})\end{array}$ \\
\hline ORF12 & 4 & 2 & $103(\mathrm{~A} / \mathrm{G}), \mathbf{1 0 2}(\mathrm{C} / \mathrm{T})$ \\
\hline ORF20 & 12 & 4 & 131(A/G), 191(C/T), 278(A/G), 449(C/G) \\
\hline$B A 87 d 17 t 3$ & 6 & 4 & $212(\mathrm{C} / \mathrm{T}), \mathbf{2 6 2}(\mathrm{C} / \mathrm{T}), \mathbf{3 8 4}(\mathrm{A} / \mathrm{T}), \mathbf{4 4 4}(\mathrm{C} / \mathrm{T})$ \\
\hline$B A 213 c 14 t 7$ & 14 & 13 & $\begin{array}{l}78(\mathrm{C} / \mathrm{T}), \mathbf{9 6}(\mathrm{C} / \mathrm{T}), \mathbf{1 3 9}(\mathrm{A} / \mathrm{G}), \mathbf{1 5 2}(\mathrm{A} / \mathrm{T}), \mathbf{2 1 8}(\mathrm{A} / \mathrm{C}), \mathbf{2 3 6}(\mathrm{C} / \mathrm{T}), \mathbf{2 4 4}(\mathrm{C} / \mathrm{T}), \mathbf{2 5 3}(\mathrm{C} / \mathrm{T}) \\
\mathbf{2 7 4}(\mathrm{A} / \mathrm{T}), \mathbf{2 8 4}(\mathrm{A} / \mathrm{G}), \mathbf{2 8 9}(\mathrm{A} / \mathrm{G}), \mathbf{3 4 5}(\mathrm{C} / \mathrm{T}), \mathbf{3 4 7}(\mathrm{C} / \mathrm{T})\end{array}$ \\
\hline$P R K$ & 9 & - & \\
\hline GP179 & 5 & - & \\
\hline StPto & 4 & - & \\
\hline 239E4left & 9 & - & \\
\hline Total & 100 & 45 & \\
\hline
\end{tabular}

Bold values indicate the SNP numbers

${ }^{a}$ SNP numbering is according to the PoMaMo database (http://www.gabipd.org/projects/Pomamo/). New SNPs are numbered starting with the first nucleotide after the sequencing primer

represented as a mixture of all the main subgroups, so it would be impossible to partition individuals into groups.

One hundred SNPs, between four and 20 SNPs per amplicon, were scored and evaluated individually for association with the G. pallida QRL on chromosome V, by testing for unequal distribution of the SNP alleles between the HC-positive (resistant) sample population and the HC-negative (susceptible) control population. Forty-five SNPs were associated with resistance at $P<0.01$ (Table 3). The significant SNPs were restricted to six loci, namely $G P 21$, ORF3, ORF12, ORF20, BA87d17t3 and BA213c14t7 (Table 3, Fig. 2), which span a genetic distance of $2 \mathrm{cM}$.

Analysis of linkage disequilibrium between all pairs of SNP markers revealed LD among groups of SNPs, most strongly within the same amplicon. Figure 3 shows the LD matrix for the 100 SNPs. SNPs in LD to each other with $q<10^{-2}$ are shown as grey squares, whereas strong LD with $q<10^{-4}$ is represented by dark grey squares. Strong LD $\left(q<10^{-4}\right)$ between SNPs in different amplicons was observed only in the 3-cM region between GP21 and GP179 and did not extend to StPto and 239E4left, located 5 and $35 \mathrm{cM}$, respectively, distal to GP179 (Figs. 1 and 3). The SNPs were then grouped using the criterion of strong LD $\left(q<10^{-4}\right)$ between SNP pairs. The 45 SNPs associated with resistance sorted into six groups of 2-18 SNPs (Fig. 3, Table 4). Group C included 18 SNPs, which closely matched the distribution of the $\mathrm{HC}$ marker in the sample and control population. These 18 SNPs originated from loci
Fig. 2 Line diagram showing the $P$ values for association between the SNPs and nematode resistance at the ten analysed loci. Y-axis: - $\log P$. X-axis: 100 SNPs in consecutive order. The loci are shown below the $\mathrm{x}$-axis and are connected with the corresponding SNPs by broken lines. SNPs with $P \leq 0.01(-\log P \geq 2)$ were restricted to the loci GP21, ORF3, ORF12, ORF20, $B A 87 d 17 t 3$ and $B A 213 c 14 t 7$

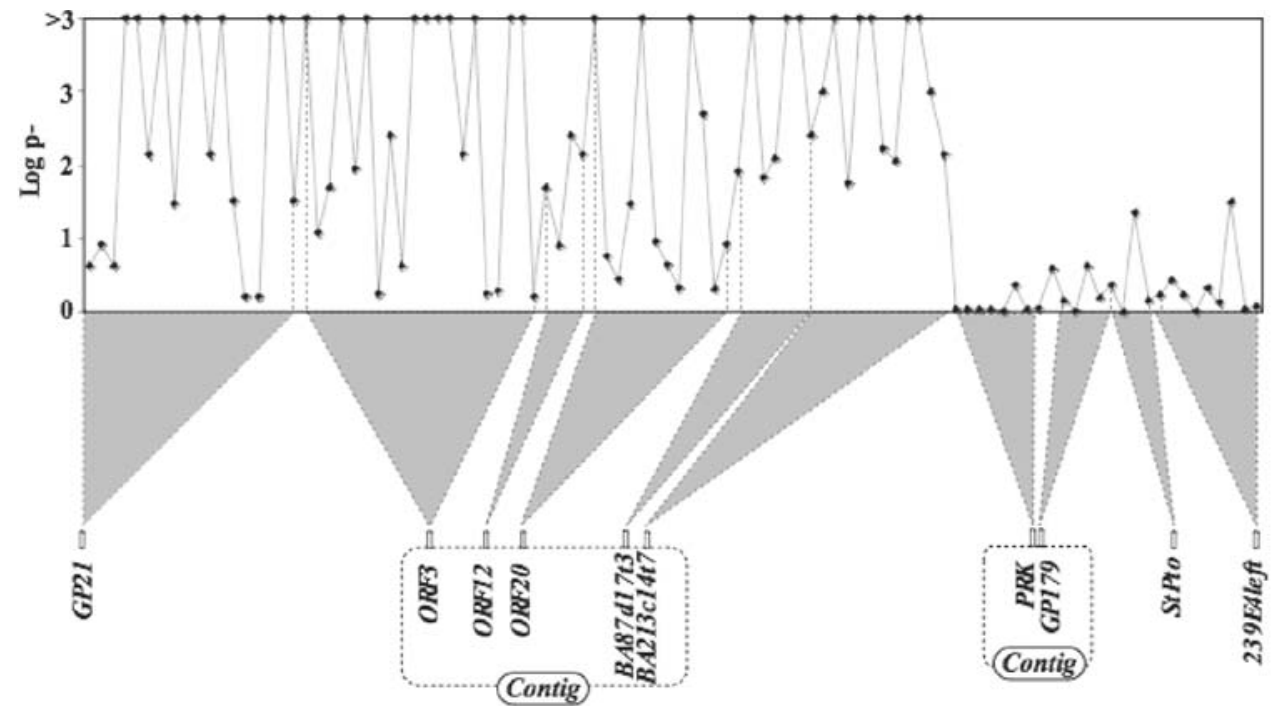


Fig. 3 Linkage disequilibrium (LD) matrix for the SNPs. The 100 analysed SNPs are plotted on both axes. SNPs physically linked within the same amplicon are framed. LD significant with $q \leq 10^{-4}$ is indicated in dark grey and LD significant with $0.01>q<10^{-4}$ is indicated in light grey. The names of the ten loci analysed are shown on top of the matrix. The LD blocks $\mathrm{C}-\mathrm{G}$ are shown on the vertical axes of the LD matrix, with the borders of LD blocks indicated by horizontal lines

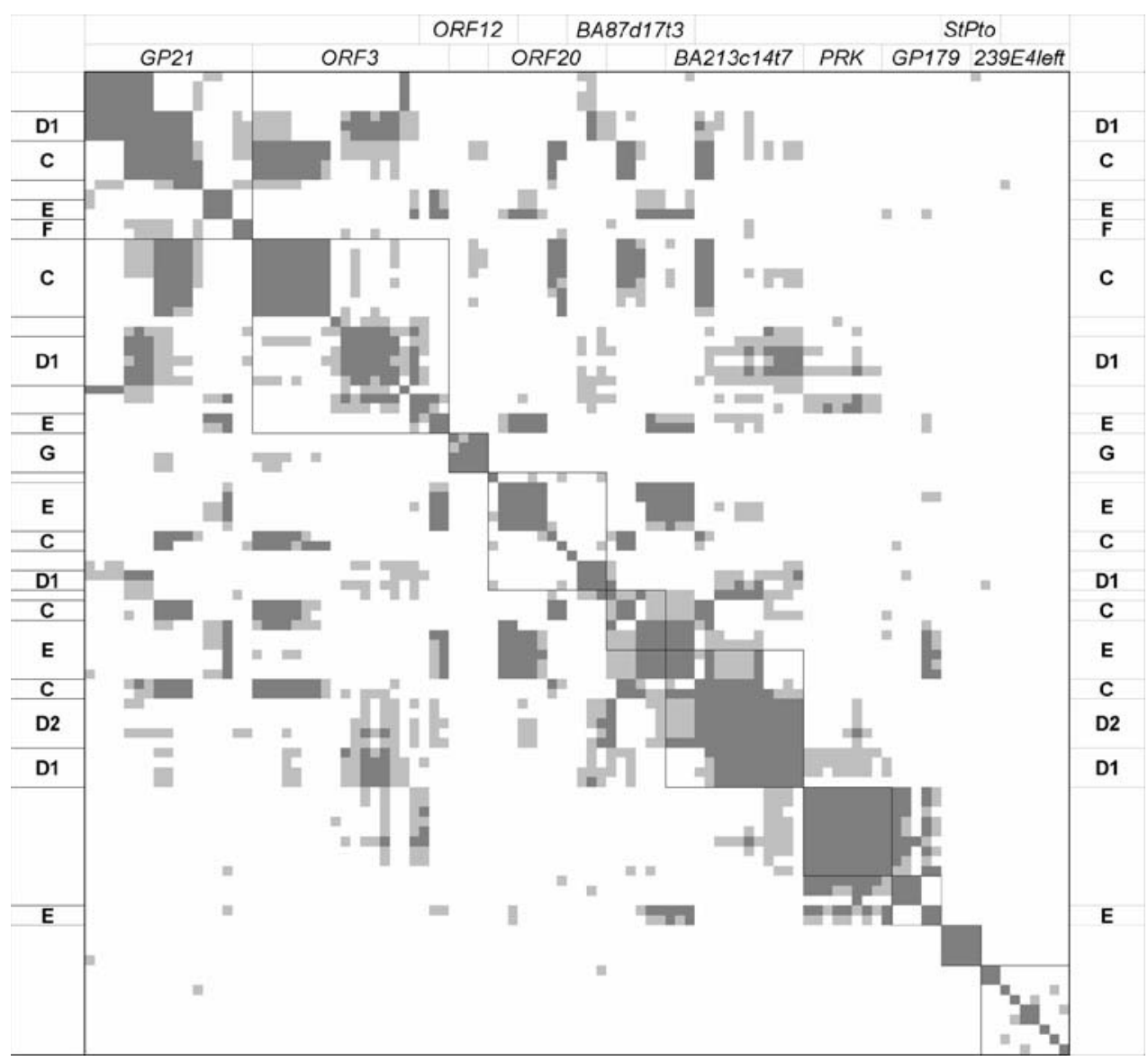

GP21, ORF3, ORF20, BA87d17t3 and BA213c14t7. All SNPs in group $C$ were associated with nematode resistance at $P=0.0001$. Three SNPs (ORF3-SNP433, ORF3SNP438, ORF3-SNP596) matched the distribution of the HC marker. Thirty-three of 41 individuals in the resistant sample population were heterozygous or homozygous for all 18 SNP alleles associated with resistance (Table 5), whereas the remaining eight individuals were homozygous for a portion of one to four of the $18 \mathrm{SNP}$ alleles associated with susceptibility (Table 5). Three individuals of the susceptible control population carried exclusively SNP alleles associated with susceptibility, whereas the remaining 35 individuals were heterozygous for one to seven of the 18 SNP alleles associated with resistance. Inspection of the 'recombinants' in both the sample and control population (susceptible individuals heterozygous for some resistance SNP alleles and resistant individuals homozygous for some susceptibility SNP alleles) did not permit positioning more precisely the major QRL, for which the $\mathrm{HC}$ marker is diagnostic, within the $2 \mathrm{~cm}$ interval GP21 - BA213c14t7.

SNP groups D1, D2, F and G were also not informative for fine mapping the QRL, D1 and D2 because all SNPs in these groups were similarly associated with resistance, $\mathrm{F}$ and $\mathrm{G}$ because each of these groups consisted only of SNPs within the same amplicon. However, LD group E including 16 SNPs at the loci GP21, ORF3, ORF20, BA87d17t3, $B A 213 c 14 t 7$ and GP179 showed an association gradient, because only four SNPs at the loci $B A 87 d 17 t 3$ and $B A 213 c 14 t 7$ were associated with resistance at $P<0.01$, whereas the remaining 12 SNPs in LD group E were not significantly associated with resistance. This peak of significant associations observed across six loci covering a genetic distance of $3 \mathrm{cM}$ suggests that one or more genes for quantitative nematode resistance are located more closely to $B A 87 d 17 t 3$ and $B A 213 c 14 t 7$ than to GP21, $O R F 3$ and $O R F 20$ on the left, or GP179 on the right.

\section{Discussion}

A population of 79 tetraploid individuals was analysed in our study, which represents a maximum of $316(4 \times 79)$ chromosome V haplotypes. To capture 316 haplotypes, a minimum of nine bi-allelic SNP markers in linkage equilibrium is required $\left(2^{9}=512\right)$. Genotyping 100 SNPs had therefore the potential to capture all chromosome V haplotypes that were actually present in the 79 individuals, at least the most common ones. The fact that most SNPs were 
Table 4 Grouping of SNPs based on LD, including the SNPs associated with nematode resistance

\begin{tabular}{|c|c|}
\hline LD group & SNP positions and significance of association with resistance ${ }^{a}$ \\
\hline $\mathrm{C}$ & $\begin{array}{l}\text { GP21_SNP1090***, GP21_SNP1165***, GP21_SNP1170***, GP21_SNP1257***, ORF3_SNP93***, } \\
\text { ORF3_SNP143***, ORF3_SNP433***, ORF3_SNP438***, ORF3_SNP472***, ORF3_SNP520***, } \\
\text { ORF3_SNP596***, ORF3_SNP611***, ORF20_SNP449***, ORF20_SNP278***, } \\
\text { BA87d17t3_SNP212***, BA87d17t3_SNP384***, BA213c14t7_SNP274***, } \\
\text { BA213c14t7_SNP139*** }\end{array}$ \\
\hline D1 & $\begin{array}{l}\text { GP21_SNP1144***, GP21_SNP1199***, GP21_SNP1274***, ORF3_SNP325*, ORF3_SNP328***, } \\
\text { ORF3_SNP380**, ORF3_SNP460***, ORF3_SNP503**, ORF20_SNP191***, } \\
\text { BA213c14t7_SNP218**, BA213c14t7_SNP253***, BA213c14t7_SNP284***, } \\
\text { BA213c14t7_SNP345** }\end{array}$ \\
\hline D2 & $\begin{array}{l}\text { BA87d17t3_SNP184*, BA213c14t7_SNP347**, BA213c14t7_SNP289***, BA213c14t7_SNP244**, } \\
\text { BA213c14t7_SNP96**, BA213c14t7_SNP78** }\end{array}$ \\
\hline $\mathrm{E}$ & $\begin{array}{l}\text { GP21_SNP1065, ORF3_SNP337, ORF3_SNP548, ORF20_SNP307, ORF20_SNP284*, } \\
\text { ORF20_SNP275, ORF20_SNP233, ORF20_SNP117, BA87d17t3_SNP222*, BA87d17t3_SNP262**, } \\
\text { BA87d17t3_SNP444***, BA213c14t7_SNP287*, BA213c14t7_SNP236**, } \\
\text { BA213c14t7_SNP152***, GP179_SNP246, GP179_SNP208 }\end{array}$ \\
\hline $\mathrm{F}$ & GP21_SNP1155**,GP21_SNP1185** \\
\hline $\mathrm{G}$ & ORF12_SNP181*, ORF12_SNP148, ORF12_SNP103**, ORF12_SNP102** \\
\hline
\end{tabular}

Table 5 The 18 SNP alleles of LD group C associated with resistance or susceptibility to G. pallida

\begin{tabular}{lll}
\hline SNPs in group C & 'Resistant' & $\begin{array}{l}\text { 'Susceptible' } \\
\text { SNP allele }\end{array}$ \\
\hline GP21-SNP1090 & $\boldsymbol{C}$ allele & $\boldsymbol{A}$ \\
GP21-SNP1165 & $\boldsymbol{C}$ & $\boldsymbol{G}$ \\
GP21-SNP1170 & $\boldsymbol{C}$ & $\boldsymbol{T}$ \\
GP21-SNP1257 & $\boldsymbol{G}$ & $\boldsymbol{T}$ \\
ORF3-SNP93 & $\boldsymbol{T}$ & $\boldsymbol{C}$ \\
ORF3-SNP143 & $\boldsymbol{C}$ & $\boldsymbol{T}$ \\
ORF3-SNP433 & $\boldsymbol{C}$ & $\boldsymbol{A}$ \\
ORF3-SNP438 & $\boldsymbol{C}$ & $\boldsymbol{G}$ \\
ORF3-SNP472 & $\boldsymbol{T}$ & $\boldsymbol{A}$ \\
ORF3-SNP520 & $\boldsymbol{G}$ & $\boldsymbol{A}$ \\
ORF3-SNP596 & $\boldsymbol{A}$ & $\boldsymbol{T}$ \\
ORF3-SNP611 & $\boldsymbol{T}$ & $\boldsymbol{A}$ or $\boldsymbol{G}$ \\
ORF20-SNP449 & $\boldsymbol{C}$ & $\boldsymbol{G}$ \\
ORF20-SNP278 & $\boldsymbol{G}$ & $\boldsymbol{A}$ \\
BA87d17t3-SNP384 & $\boldsymbol{T}$ & $\boldsymbol{A}$ \\
BA87d17t3-SNP212 & $\boldsymbol{T}$ & $\boldsymbol{C}$ \\
BA213c14t7-SNP139 & $\boldsymbol{A}$ & $\boldsymbol{G}$ \\
BA213c14t7-SNP274 & $\boldsymbol{T}$ & $\boldsymbol{A}$ \\
\hline
\end{tabular}

a Three-allelic SNP

in LD with one or more other SNPs also indicated that the SNP genotyping approached saturation. The 100 SNPs were distributed over ten loci. SNPs closely associated with resistance to $G$. pallida were restricted to the six loci distal to the marker locus GP179, spanning a genetic distance of $2 \mathrm{cM}$. The physical size of this region is around $1.7 \mathrm{Mb}$
(Achenbach et al. manuscript in preparation). This region could be even larger, as no further marker was available for SNP analysis distal to GP21. The association analysis delimited therefore the genomic region harbouring the $\mathrm{QRL}$ at one side to the interval $B A 213 c 14 t 7-G P 179$, but not at the other.

Due to the heterozygocity and tetraploidy of the cultivated potato, the inference of specific haplotypes from SNP data is not straightforward. The SNP markers are unphased and the SNP allele dosage has to be considered in haplotype models (J. Neigenfind et al. 2008). Instead of haplotype models we used the strong LD observed among several SNPs to distinguish six LD groups, which included the SNPs associated with nematode resistance. The six groups discussed here represent only a subset of all LD groups present in the population, as LD groups comprising only SNPs not associated with nematode resistance were not considered and rare LD groups were not detected. Several different groups of SNPs in LD with each other were found, each group including SNPs associated with resistance to G. pallida. This indicates the existence of multiple alleles either at a single resistance locus or at several, physically closely linked loci, which control the quantitative resistance to G. pallida in this particular genomic region on potato chromosome V.

The most prominent resistance allele was associated with LD group $\mathrm{C}$, which closely correlated with the $\mathrm{HC}$ marker. This is not surprising, as presence or absence of the $\mathrm{HC}$ marker was the criterion for selecting resistant and susceptible individuals in the population. No association gradient was observed within LD group C. The 18 SNPs in group $\mathrm{C}$ were all associated with resistance with similar 
strength and defined a large haplotype block comprising at least $2 \mathrm{cM}$ or more than $400 \mathrm{kbp}$. Positioning the resistance gene(s) more precisely within this haplotype block was not possible based on the available SNP data. In the case of LD group $\mathrm{C}$, two contrasting haplotype models could be deduced from the nucleotide alleles of the 18 SNP markers, one diagnostic of resistance similar to the HC marker, and the other one of susceptibility. The resistant haplotype was dominant. All heterozygous individuals having at least one dose of this resistant haplotype were resistant to G. pallida, whereas none of the susceptible individuals carried this haplotype. The gene(s) for resistance to G. pallida pathotype $\mathrm{Pa} 2 / 3$ associated with the $\mathrm{HC}$ marker, together with the SNP alleles in the resistant haplotype, have been introgressed into the European potato breeding pool (Solanum tuberosum) from the wild potato species Solanum vernei (Sattarzadeh et al. 2006). Large haplotype blocks may have been preserved due to limited recombination events in the breeding history of the germplasm analysed in our study. The pedigree information for $G$. pallida resistant varieties that is available from the 'Potato Pedigree database' (van Berloo et al. 2007, http://www.plantbreeding.wur.nl/potatopedigree/) is partial and contains 'dead ends'. A number of $S$. vernei clones, which do not exist anymore, have been used several times in the pedigrees. The historical records do not allow identifying precisely the $S$. vernei resistance source and the number of meiotic generations, which separate contemporary germ plasm from the original introgressions. In general, variety pedigrees that can be traced back through the last 100 years include less than 10 meiotic generations (Love 1999; Gebhardt et al. 2004; van Berloo et al. 2007). Reduced recombination between homeologous chromosomes caused, for example, by inversions in the introgressed $S$. vernei segment relative to $S$. tuberosum, may also have contributed to the maintenance of a large haplotype block in the breeding populations. The existence of a 70-kbp inversion between S. demissum and S. tuberosum in this genome segment has been demonstrated (Ballvora et al. 2007). Suppression of recombination depending on the source and size of the introgression from wild species has been shown in tomato (Liharska et al. 1996; Kaloshian et al. 1998). Linkage disequilibrium extending over several centimorgan has also been observed by Simko et al. (2006) and $\mathrm{Li}$ et al. (2008) in different populations of tetraploid and diploid potato genotypes.

In contrast to $\mathrm{LD}$ group $\mathrm{C}$, a peak of marker trait associations was observed within LD group E comprising 16 SNPs at 6 loci spanning $3 \mathrm{cM}$. Here, four SNPs at the loci $B A 87 d 17 t 3$ and BA213c14t7 were associated with nematode resistance, whereas the SNPs at the flanking loci were not any more associated with nematode resistance. With the assumption that the QRL is controlled by one gene with multiple alleles, the association gradient observed in LD group E places this gene within or physically close to the genomic region delimited by BACs BA87d17 and $\mathrm{BA} 213 \mathrm{c} 14$. This region harbours the $R l$ resistance gene and two paralogous members of the $R l$ gene family (Ballvora et al. 2007). When the alternative model is true, meaning that several genes in this genomic region control nematode resistance, at least one of these genes should be located within or physically close to the genomic region delimited by BACs BA87d17 and BA213c14. Association mapping in a large population of individuals lacking the almost qualitative HC marker would be of interest to better resolve the genetic architecture of the QRL based on weaker resistance alleles.

The individuals analysed in this study have been generated by inter-crossing tetraploid, highly heterozygous parents. LD decays more rapidly in outcrossing species when compared to selfing species, where recombination has no consequence for the genotype of homozygous individuals (Nordborg 2000). In potato, the diminishing effect of outcrossing on LD is counteracted by the vegetative propagation, which reduces the number of meiotic generations separating individual breeding clones and varieties (Gebhardt et al. 2004; van Berloo et al. 2007). The observed phenotypic and genotypic variability not only results from recombination, but also from a larger number, when compared with diploids, of chromosomal haplotype combinations that are possible in $4 \mathrm{x}$ by $4 \mathrm{x}$ crosses with tetrasomic inheritance. This might be one reason for the large genetic distances of similar size, which usually separate tetraploid individuals despite their relatedness by pedigree, and thereby the lack of population structure ( $\mathrm{Li}$ et al. 2005). Another reason for lack of population structure might be an underlying uniformity of the genetic material due to its selective adaptation for general agronomic performance. Populations of varieties and breeding clones are highly suitable therefore to detect associations between markers and agronomic traits such as nematode resistance, due to the existence of large haplotype blocks and lack of population structure in this material. These properties make such populations less suitable for candidate gene verification due to lack of genetic resolution. Candidate genes for the QRL on potato chromosome V are, among other genes (Ballvora et al. 2007), the members of the $R I$ gene family. However, for identifying among all possible candidates the causal gene(s) for the observed nematode resistance phenotype in this genomic region, the full genome sequence of potato (http://www. potatogenome.net/) or the highly co-linear tomato (http:// www.sgn.cornell.edu/about/tomato_sequencing.pl) will be needed, together with highly effective experimental systems for functional analysis of candidate genes. 
Acknowledgments This work was supported by grant $0313114 \mathrm{~A}$ (GABI-CONQUEST project) of the German Federal Ministry for Education and Research (BMBF) in the framework of the GABI (genome analysis in the biological system plant) program.

Open Access This article is distributed under the terms of the Creative Commons Attribution Noncommercial License which permits any noncommercial use, distribution, and reproduction in any medium, provided the original author(s) and source are credited.

\section{References}

Bakker E, Achenbach U, Bakker J, van Vliet J, Peleman J, Segers B, van der Heijden S, van der Linde P, Graveland R, Hutten R, van Eck H, Coppoolse E, van der Vossen E, Bakker J, Goverse A (2004) A high-resolution map of the Hl locus harbouring resistance to the potato cyst nematode Globodera rostochiensis. Theor Appl Genet 109:146-152

Ballvora A, Jöcker A, Viehöver P, Ishihara H, Paal J, Meksem K, Bruggmann R, Schoof H, Weisshaar B, Gebhardt C (2007) Comparative sequence analysis of Solanum and Arabidopsis in a hot spot for pathogen resistance on potato chromosome $\mathrm{V}$ reveals a patchwork of conserved and rapidly evolving genome segments. BMC Genom 8:112. doi:10.1186/1471-2164-8-112

Benjamini Y, Krieger A, Yekutieli D (2005) Two staged linear step up FDR controlling procedure. Unpublished report, available at http://www.math.tau.ac.il/ ybenja

Bormann CA, Rickert AM, Castillo Ruiz RA, Paal J, Lübeck J, Strahwald J, Buhr K, Gebhardt C (2004) Tagging quantitative trait loci for maturity-corrected late blight resistance in tetraploid potato with PCR-based candidate gene markers. Mol Plant Microbe Interact 17:1126-1138

Corpet F (1988) Multiple sequence alignment with hierarchical clustering. Nucleic Acids Res 16:10881-10890

Caromel B, Mugniery D, Lefebvre V, Andrzejewski S, Ellisseche D (2003) Mapping QTLs for resistance against Globodera pallida (stone) $\mathrm{Pa} 2 / 3$ in a diploid potato progeny originating from Solanum spegazzinii. Theor Appl Genet 106:1517-1523

Feingold S, Lloyd J, Norero N, Bonierbale M, Lorenzen J (2005) Mapping and characterization of new EST- derived microsatellites for potato (Solanum tuberolsum L.). Theor Appl Genet 111:456-466

Gebhardt C, Ballvora A, Walkemeier B, Oberhagemann P, Schüler K (2004) Assessing genetic potential in germplasm collections of crop plants by marker-trait association: a case study for potatoes with quantitative variation of resistance to late blight and maturity type. Mol Breed 13:93-102

Gebhardt C, Valkonen JPT (2001) Organization of genes controlling disease resistance in the potato genome. Annu Rev Phytopathol 39:79-102

Greco N (1988) Potato cyst nematodes: Globodera rostochiensis and G. pallida. Nematology circular no. 149. Florida Department of Agriculture and Consumer Services, Division of Plant Industry, Gainesville

Kaloshian I, Yaghoobi J, Liharska T, Hontelez J, Hanson D, Hogan P, Jesse T, Wijbrandi J, Simons G, Vos P, Zabel P, Williamson VM (1998) Genetic and physical localization of the root-knot nematode resistance locus $\mathrm{Mi}$ in tomato. Mol Gen Genet 257:376-385

Kort J, Ross H, Rumpenhorst HJ, Stone AR (1977) An international scheme for the identifying and classifying pathotypes of potato cyst- nematodes Globodera rostochiensis and G.pallida. Nematologica 23:333-339

Kreike CM, De Koning JRA, Vinke JH, Van Ooijen JW, Stiekema WJ (1994) Quantitatively-inherited resistance to Globodera pallida is dominated by one major locus in Solanum spegazzinii. Theor Appl Genet 88:764-769

Leister D, Ballvora A, Salamini F, Gebhardt C (1996) A PCR based approach for isolating pathogen resistance genes from potato with potential for wide application in plants. Nat Genet 14:421-429

Liharska TB, Koornneef M, van Wordragen M, van Kammen A, Zabel $\mathrm{P}$ (1996) Tomato chromosome 6: effect of the alien chromosomal segment on recombinant frequencies. Genome 39:485-990

Li L, Strahwald J, Hofferbert HR, Lübeck J, Tacke E, Junghans H, Wunder J, Gebhardt C (2005) DNA variation at the invertase locus invGE/GF is associated with tuber quality traits in populations of potato breeding clones. Genetics 170:813-821

Li L, Paulo M-J, Strahwald J, Lübeck J, Hofferbert H-R, Tacke E, Junghans H, Wunder J, Draffehn A, van Eeuwijk F, Gebhardt C (2008) Natural DNA variation at candidate loci is associated with potato chip color, tuber starch content, yield and starch yield. Theor Appl Genet 116:1167-1181

Love SL (1999) Founding clones, major contributing ancestors and exotic progenitors of prominent American potato cultivars. Am J Potato Res 76:263-272

Martin GB, Brommonschenkel SH, Chunwongse J, Frary A, Ganal MW, Spivey R, Wu T, Earle ED, Tanksley SD (1993) Map-based cloning of a protein kinase gene conferring disease resistance in tomato. Science 262:1432-1435

Meksem K, Leister D, Peleman J, Zabeau M, Salamini F, Gebhardt C (1995) A high resolution map of the vicinity of the $R l$ locus on chromosome $\mathrm{V}$ of potato based on RFLP and AFLP markers. Mol Gen Genet 249:74-81

Meyer S, Nagel A, Gebhardt C (2005) PoMaMo: a comprehensive database for potato genome data. Nucleic Acids Res 33:D666D670

Milbourne D, Meyer RC, Collins AJ, Ramsay LD, Gebhardt C, Waugh $\mathrm{R}$ (1998) Isolation, characterisation and mapping of simple sequence repeats. Mol Gen Genet 259:233-245

Mugniéry D, Phillips MS (2007) The nematode parasites of potato. In: Vreudgenhil D, Bradshaw J, Gebhardt C, Govers F, MacKerron DKL, Taylor MA, Ross HA (eds) Potato biology and biotechnology advances and perspectives. Elsevier, Amsterdam, pp 569591

Neigenfind J, Gyetvai G, Basekow R, Diehl S, Achenbach U, Gebhardt C, Selbig J, Kersten B (2008) Haplotype inference from unphased SNP data in heterozygous polyploids based on SAT. BMC Genomics 9:356

Niewöhner J, Salamini F, Gebhardt C (1995) Development of PCR assays diagnostic for RFLP marker alleles closely linked to alleles Grol and H1, conferring resistance to the root cyst nematode Globodera rostochiensis in potato. Mol Breed 1:65-78

Nordborg M (2000) Linkage disequilibrium, gene trees and selfing: an ancestral recombination graph with partial self- fertilization. Genetics 154:923-929

Pritchard JK, Stephens M, Donelly P (2000) Inference of population structure using multilocus genotype data. Genetics 155:945-959

Rickert AM, Kim JM, Meyer S, Nagel A, Ballvora A, Oefner PJ, Gebhardt C (2003) First-generation SNP/InDel markers tagging loci for pathogen resistance in the potato genome. Plant Biotechnol J 1:399-410

van der Rouppe Voort J, Lindeman W, Folkertsma R, Hutten R, Overmars H, van der Vossen E, Jacobsen E, Bakker J (1998) A QTL for broad-spectrum resistance to cyst nematode species (Globodera spp.) maps to a resistance gene cluster in potato. Theor Appl Genet 96:654-661

van der Rouppe Voort J, van der Vossen E, Bakker E, Overmars H, van Zandvoort P, Hutten R, Klein Lankhorst R, Bakker J (2000) Two additive QTLs conferring broad-spectrum resistance in potato to Globodera pallida are localized on resistance gene clusters. Theor Appl Genet 101:1222-1230 
Sattarzadeh A, Achenbach U, Lübeck J, Strahwald J, Tacke E, Hofferbert HR, Rothsteyn T, Gebhardt C (2006) Single nucleotide polymorphism (SNP) genotyping as basis for developing a PCR-based marker highly diagnostic for potato varieties with high resistance to Globodera pallida pathotype Pa2/3. Mol Breed 18:301-312

Simko I, Haynes KG, Jones RW (2006) Assessment of linkage disequilibrium in potato genome with single nucleotide polymorphism markers. Genetics 173:2237-2245
Van Berloo R, Hutten RCB, van Eck HJ, Visser RGF (2007) An online potato pedigree database resource. Potato Res 50:45-57

Tenesa A, Knott SA, Carothers AD, Visscher PM (2003) Power of linkage disequilibrium mapping to detect a quantitative trait locus (QTL) in selected samples of unrelated individuals. Ann Hum Genet 67:557-566 